\title{
Free Quality PDA Software for Physicians
}

\author{
Jie Li
}

\begin{abstract}
As PDAs are becoming practical tools for health care professionals, medical librarians are frequently asked to give recommendations of PDA software. Based on recommendations from medical librarians, physicians, and medical students, an annotated list of free PDA software has been compiled to meet the needs of medical librarians. The list includes software for Palm OS and Pocket PC and is divided into sections based on the software's function. [Article copies available for a fee from The Haworth Document Delivery Service: 1-800-HAWORTH. E-mail address: <docdelivery@haworthpress.com>Website: 〈http://www.HaworthPress.com> (C) 2004 by The Haworth Press, Inc. All rights reserved.]
\end{abstract}

KEYWORDS. PDA, personal digital assistant, handheld computers, medical software, handheld computing

\section{INTRODUCTION}

Rapid development in information technology has made Personal Digital Assistants (PDAs), also called handheld devices or handheld computers, a practical tool for health professionals. PDAs, with a wide range of evidence sources installed, may be used as support tools for clinical decision-making at the point of care, for evidence-based learning and practicing, and for physician performance improvement. 1,2 According to a Manhattan Research study, approximately 35\% of practicing

Jie Li, MLS, AHIP (jli@jaguar1.usouthal.edu) is Coordinator of Medical Center Site, Biomedical Library, University of South Alabama, 2451 Fillingim Street, Mobile, AL 36617.

Journal of Electronic Resources in Medical Libraries, Vol. 1(1) 2004

http://www.haworthpress.com/store/product.asp?sku=J383

02004 by The Haworth Press, Inc. All rights reserved. 
physicians were actively using a PDA in $2002 .{ }^{3}$ Medical librarians are becoming more involved with PDAs and PDA software as part of their role in medical education. Librarians are frequently contacted for recommendations for PDA software and hardware selections. Many health science libraries provide seminars and workshops discussing PDA hardware and software and its usage in patient care.

There are two major operating systems for PDA, Palm operating system (Palm OS) and Pocket PC. Palm OS was the first operating system for the PDA, so there is more software developed for Palm OS, especially free software developed by physicians and medical residents. In the past several years, more software has been developed for the Pocket PC. However, Palm OS is still more popular in the medical community as it requires less memory than the Pocket PC.

Based on recommendations from medical librarians, physicians, and medical students, an annotated list of free PDA software has been compiled to meet the needs of medical librarians. Every Palm OS compatible software in the list has been downloaded and tested. Only free PDA software that was judged by the author as a quality tool is included. The list is far from inclusive. More medical software for the PDA, free or for a fee, may be found in the Web sites listed in the Appendix.

\section{DRUG DATABASES}

\section{eDrugs Software <http://www.geocities.com/fhirning/eDrugsSoftware.html/>}

eDrugs software programs are drug information programs for PDAs, written by Fred C. Hirning, PharmD, FAPhA. There are two basic programs: eDrugsData and eDrugsRenal. eDrugsData contains information on over 2,450 generic drug listings. It contains over 12,000 drug names and dosage forms. eDrugsRenal lists over 560 different drugs and has over 2,500 dosage adjustment recommendations and contains recommended dosage adjustments for patients with renal impairments. Multiple recommendations are listed when available. Both databases give the generic name, trade name, use or class, dosage form, usual dose, pregnancy risk factor, and additional information for each listing. Users may add their frequently used drugs to the list. All fields are searchable. Each of the programs is available in HanDBase < http://www. ddhsoftware.com/handbase.html>, MobileDB Lite <http://mobiledb.com/>, JFile 5.0 and JFile $4.0<$ http://www.land-j.com/jfile.html> formats for Palm OS and HandBase format for Pocket PC. The respective database 
program must be installed onto the PDA in order to open the eDrugs program. All of the above database programs, except JFile 4.0, are not free. eDrugsData uses $231 \mathrm{~KB}$ of memory and eDrugsRenal uses $78 \mathrm{~KB}$ memory. Last update, November 25, 2002.

\section{ePocrates Rx} <http://epocrates.com/products/rxPro6/prod_diff.cfm>

ePocrates Rx, a highly recommended free clinical drug database, contains peer-reviewed information about more than 2,700 brand and generic drugs, including off-label indications and formularies in an easy-to-use format. The database is searchable by the drug's generic name, trade name, or class. For each drug, more information is available under tabs labeled "Adult Dosing," "Peds Dosing," "Contraindications/Cautions," "Drug Interactions," "Adverse Reactions," "Manufacturer/Cost Info," and "Other Info." There is also a "Notes" feature for entering personal information from personal observations. Under "MultiCheck," up to 30 drugs at a time may be selected for checking drug-drug interactions. Graffiti or the built-in keyboard may be used to enter drug names for searches. ePocrates Rx comes with an AutoUpdate feature. Every time a hotsync is performed, it automatically connects to the server to download update information. A DocAlert provides the latest updated information about drugs. Insurance companies' co-pay information is also available. Clinical tables and guidelines, drug interactions with alternative medicines, and the ePocrates ID infectious disease guide are only available for those who purchase the premium ePocrates Rx Pro version. Available for Palm OS only, and requires 2.5 MB of memory. Last update, Version 6.0, December 5, 2002.

\section{Johns Hopkins Antibiotic Guide <http://hopkins-abxguide.org/>}

The Johns Hopkins Point of Care Information Technology (POC-IT) Antibiotic (ABX) Guide is a handheld-ready adaptation of the online ABX Guide. It is optimized for use at the point of care. The ABX Guide is an electronic reference tool that provides continually updated and easily accessible digests of the most current expert opinions and guidelines for the diagnosis and treatment of infectious diseases. The ABX Guide has three categories: Diagnosis, which lists diagnosis for nineteen systems; Pathogen, which includes bacteria, fungi, parasites, viruses, and other; and Antibiotic, which includes antibacterial, antifungal, 
antimycobacterial, antiparasitic, antivirual, and other. Any of the categories may be easily accessed by tapping on the tabs on the right of the screen. Antibiotics are listed by their generic names with a screen of four columns listing brand name, drug forms, dosages, and costs. Other display information includes class, indications, usual adult dosing, adverse reactions, drug interactions, comments, glomerular filtration, pregnancy risk, and author. The built-in function makes it easy to move it from handheld device to memory card. It connects to a server to get updated information whenever a hotsync is performed. However, it lacks pediatric data, and the disease database is not as extensive as other for-fee infectious disease drug database software. The Guide is available for all forms of PDAs and uses $1363 \mathrm{~KB}$ of memory. Last update, Version 2.0, 2002.

\section{mobilePDR <http://www.pdr.net/>}

mobilePDR is the concise version of the Physician's Desk Reference $(P D R)$ for handheld devices. mobilePDR displays a brief summary, extracted from $P D R$, of drug indications, contraindications, warnings and precautions, adult and pediatric dosing, adverse reactions, drug-drug interactions, therapeutic class, Black Box warnings, Drug Enforcement Agency (DEA) class, pregnancy and nursing information, and manufacturer's name. The database lists brand and generic names and may be sorted by indications or therapeutic class. It is searchable by drug's brand and generic names. Each drug has a page number referring to the print $P D R$. The latest updates of drug information are provided when hotsync is performed. Available for both Palm OS and Pocket PC and requires Palm OS 3.0 or higher and Pocket PC OS 3.0 or higher. It requires 5.4 $\mathrm{MB}$ of free memory for Palm and $9 \mathrm{MB}$ for Pocket PC. Version 1.0 was released in 2002. Only physicians, physician assistants, and nurse practitioners with valid DEA numbers are allowed to download the software.

\section{MEDICAL CALCULATORS}

\section{BreastCa}

<http://smi-web.stanford.edu/people/pcheng/breastca/index.html >

Developed by the author of MedMath, Phillip Cheng, BreastCa is a breast cancer risk prediction tool for PDAs. In particular, it is designed to be a fast, easy-to-use, and accurate implementation of two commonly-used models of breast cancer risk prediction: the Gail model 
<http://bcra.nci.nih.gov/brc/>, the most widely used breast cancer risk prediction and the basis of the National Cancer Institute (NCI) "Risk Disk"; and the Claus model, which focuses exclusively on family history risk factors. Users may select the values by tapping the down arrow key on the screen to calculate. Uses $30 \mathrm{~KB}$ of memory and is available for Palm OS only. Version 1.0 was released August 12, 2001.

\section{DietCalc \\ <http://pbrain.hypermart.net/files/dietcalc.zip>}

DietCalc is a simple program for calculating resting and active metabolic rates (RMR and AMR), which can be used to estimate daily caloric intake, ideal body weight, and body mass index all in one convenient place. These calculations are based on the Harris-Benedict Equation. "NSBRuntime.prc," which may be downloaded in the same zip file, is required in order for DietCalc to function. Available on Palm OS only and uses 115 KB of memory. Last update, Version 1.5, November 28, 2002.

\section{EBM Calculator <http://www.cebm.utoronto.ca/palm/ebmcalc/>}

EBM (Evidence-based Medicine) Calculator is designed to calculate relevant statistics for diagnostic studies, prospective studies, case control studies, randomized control trials (RCT), and conversion from odds ratio (OR) to number needed to treat (NNT). MathLib is required to run EBM Calculator. Requires $51 \mathrm{~KB}$ of memory and is available for both Palm OS and Pocket PC. Last update, August 13, 2001.

\section{ICUmath <http://www.freewarepalm.com/medical/icumath.shtml>}

ICUmath, developed by Terry Fagan, MD, includes 47 adult ICU applications that assist with 75 medical equations including pulmonary, cardiology, pharmacokinetic drug dosing, renal, electrolytes, nutrition, total parenteral nutrition (TPN), biostatistics, unit conversions, rules of thumb, plus a loan calculator. Entering data is easy using the onscreen keypad. This program needs to use the free mathLib.prc file, downloadable in the same zip file. MathLib.prc may also be downloaded for free at the MathLib Information Web page <http://www.radiks.net/\%7Erhuebner/ mathlib.html>. Requires $344 \mathrm{~KB}$ of memory and is available on Palm OS only. Last update, November 9, 2002. 


\section{MedCalc <http://medcalc.med-ia.net/>}

MedCalc is a full-featured free medical calculator for PDA. Developed by Mathias Tschopp, MD, it is designed for rapid calculation of common equations used in internal medicine. This invaluable tool for physicians, medical residents, and students is easy-to-use and comprehensive, with 75 formulas listed alphabetically and sorted by category. Users may select a custom category, as well. It may be calculated by either Système Internationale (S.I.) or United States (U.S.) units. Bibliographic references and clinical-use tips may be found for most formulas by tapping the "i" at the top right corner. Calculations for a particular patient may be stored with a time and date stamp. Multiple values may be stored. English, French, and Spanish versions are available. Only available for Palm OS and requires Palm OS 3.1 or later version. MedCalc uses $252 \mathrm{~KB}$ of memory. Last update, Version 4.2, September 13, 2002.

\section{MedMath}

\section{<http://smi-web.stanford.edu/people/pcheng/medmath/index.html>}

MedMath was developed by Philip Cheng, MD, for rapid calculation of common formulas in adult internal medicine. Although it does not have as many formulas as MedCalc does, the 34 formulas are the ones used most often by internal medicine physicians, medical residents, and students. Different categories of formulas are displayed by tapping at the pop-up trigger at the top right corner of the screen. The program comes with selectable U.S. and S.I. units. The onscreen numeric keypad with a backspace button and fast, easy-to-punch interface is a most welcome feature. Information screens provide tips on equation usage and references. The program keeps track of the last 10 equations used, and no external programs or libraries are required. Available for Palm OS only and runs on all Palm platforms. Requires $40 \mathrm{~KB}$ of memory. Last update, Version 1.21, July 7, 2002.

\section{MEDICAL APPLICATIONS}

\section{ABG Pro <http://www.stacworks.com/>}

Developed by Wade Carlson, MD, ABG Pro is a simple program that will completely analyze arterial blood gases. The program not only pro- 
vides information on metabolic or respiratory acidosis or alkalosis, but also calculates expected $\mathrm{PCO}_{2}$ and expected $\mathrm{HCO}_{3}$ when needed, and alerts if there is a concominant acid-base disorder. It will even calculate Anion Gap and Delta-Delta if necessary. "Normal ABG Values" is stated if all values are normal. The screen keypad makes it easy-to-use. Available on Palm OS only and only needs 19 KB of memory. Last update, Version 2.2, October 23, 2000.

\section{MedRules}

<http://pbrain.hypermart.net/medrules.html>

MedRules is an application featuring 40 useful clinical prediction rules taken from the medical literature. Medical rules are listed alphabetically and by category. Tap or check a box to select a value range to calculate the risks. Complete references for each rule may be found by clicking the question mark icon on the upper-right corner of the screen. MedRules was written using the NS BASIC runtime module, and the freeware NSBRuntime.prc and MathLib.prc are required. The intention of the application is for educational use only. MedRules and its libraries require $343 \mathrm{~KB}$ of memory. Last update, Version 3.01, November 28, 2002.

\section{My Patient}

<http://www.freewarepalm.com/medical/mypatients.shtml>

This application allows users to track in-patients, labs, beam checkout, and print (with printboy or other print application). Available for Palm OS only, requires Palm III or higher, uses $40 \mathrm{~KB}$ of memory. Last update, June 26, 2002.

\section{Patient Tracker by Handheldmed <http://www.patienttracker.com/download_ptpalm.htm>}

Patient Tracker allows mobile access to patient records including patient demographics, laboratory results, medication/allergy lists, test results, and radiology reports. Patient Tracker includes the ability to print notes, checkout lists, and a patient log to any IrDA enabled printer. Available for both Palm OS and Pocket PC and takes 178 KB of memory. Last update, Version 5.11, September 27, 2002. 


\section{Peds Omnibus \\ <http://palmgear.com/software/showsoftware.cfm?sid= 55690120030110120110\& prodID=43121>}

This application calculates oral medication doses, intravenous medication doses, pediatric daily values, and neurological intensive care unit (NICU) daily values; tracks patients; and has developmental milestones, growth charts, and immunization schedules. Available on Palm OS only, uses $186 \mathrm{~KB}$ of memory, and requires Palm III or higher. Last update, August 2, 2002.

\section{STAT Cardiac Clearance <http://www.statcoder.com/cardiac1.htm>}

This application guides clinicians through the complex algorithms established by the American College of Cardiology/American Heart Association Task Force on Practice Guidelines and the American College of Physicians for evaluating patients prior to noncardiac surgery with detail and explanatory footnotes. It provides a checklist that needs to be considered prior to surgery. Available on Palm OS only, and uses $97 \mathrm{~KB}$ of memory. Last update, Version 1.1, March 2002.

\section{STAT Cholesterol <http://www.statcoder.com/cholesterol.htm>}

This application guides clinicians through the Third Report of the National Cholesterol Education Program (NCEP) Expert Panel on Detection, Evaluation, and Treatment of High Blood Cholesterol in Adults (Adult Treatment Panel III or ATP III) cholesterol guidelines. It calculates absolute risk of myocardial infarction using the point method outlined in the ATP III Executive Summary <http://www.nhlbi.nih.gov/ guidelines/cholesterol/atp_iii.htm>. The calculation of risk for any symptomatic coronary heart disease using the 1998 Framingham method is available. All input is via checkboxes and trigger buttons. No graffiti writing is required. It uses an easy point-and-click interface with the option of using S.I. units. Normal values are provided. Available on Palm OS only and takes $62 \mathrm{~KB}$ of memory. Last update, Version 1.01, 2001. 


\section{STAT Growth Charts \\ <http://www.statcoder.com/growthcharts.htm>}

This program calculates growth percentiles based on the June 2000 revision of the Centers for Disease Control and Prevention (CDC) Growth Charts. It includes new body mass index-for-age charts. The age in years and months can be entered using the pop-up lists. The weight, height, and head circumference can be entered using graffiti, and sex can be selected. Metric or English units are available. If head circumference (HC) is included, HC-Age percentile is presented. Available for Palm OS only and uses $182 \mathrm{~KB}$ of memory. Last update, Version 2.07, 2002.

\section{REFERENCES}

\section{General References}

\section{EKG Reference}

\section{<http://pbrain.hypermart.net/medfiles.html>}

This is a quick reference to aid in interpreting EKGs. A doc reader is required. Available for Palm OS only, uses $3 \mathrm{~KB}$ of memory, released June 7, 2000.

\section{Endocarditis Prophylaxis <http://www.meistermed.com/MoreMeister/endocarditis_ prophylaxis.htm>}

This Guide to Endocarditis Prophylaxis Decision Making is based on the American Heart Association's "Prevention of Bacterial Endocarditis" guidelines published in JAMA 277(1997): 1794-1801. It answers the questions of whether the patient's heart condition requires endocarditis prophylaxis, whether the patient with "mitral valve prolapse" needs endocarditis prophylaxis, whether the patient who is about to undergo fiberoptic brochoscopy procedure requires prophylaxis, how long before the prophylaxis procedure the patient is supposed to take Amoxicillin, and what is the recommended prophylaxis regimen when the patient is allergic to penicillin and needs antibiotic prophylaxis before his cystoscopy. The document is formatted with hyperlinks for ideal viewing in Isilo reader. Available for Palm OS and Pocket PC. Uses 14 KB of memory. Late update, May 21, 2002. 


\section{Herbal Reference Guide <http://pbrain.hypermart.net/medfiles.html>}

This guide is based on the pharmacy continuing education module called Popular Herbs in the U.S. Market: Therapeutic Monographs $<\mathrm{http}: / / \mathrm{ww}$ w.herbalgram.org>. It is a quick reference guide to common herbal remedies. A doc reader is needed. Available for Palm OS only, uses $8 \mathrm{~KB}$ of memory. Released June 7, 2000.

\section{ICD-9 Coding Tools From Family Practice Management (FPM) <http://www.aafp.org/fpm/icd9.html>}

Family Practice Management offers a short and a long list of ICD-9 coding tools free for Palm-compatible handheld computers on doc file. The short list of about 600 codes has been a popular FPM resource over the past few years. It uses problem-oriented descriptors of conditions common in family practice to sort the ICD-9 list. The long list of about 1,500 codes common in family practice expands the short list to include a wider range of four-digit and five-digit codes. It also includes more codes useful for hospital care. Both lists are downloadable on the PC and Mac. To view doc files, a user will need a "doc reader" such as AportisDoc <http://www.aportis.com/>, Quickword <http://www.cesinc.com/> or TealDoc <http://www.tealpoint.com/> to display the doc files. AportisDoc is free, but the other two are not. Available for Palm OS only. The short list uses $14 \mathrm{~KB}$, and the long list uses $52 \mathrm{~KB}$ of memory. Last update, 2002.

\section{JournalToGo <http://www.journaltogo.com/>}

JournalToGo provides weekly updated journal abstracts from the National Library of Medicine and news articles from Reuters to PDA by Health Tech Solutions. Every time a hotsync is performed, it automatically accesses the JournalToGo's Web site to get the most current information. Sign up is required for the free service. Users have the option to copy the documents to memo pad and to e-mail them to a friend if the PDA has an Internet connection. Journal citations and abstracts provided are updated as they are published. Available for both Palm OS and Pocket PC. Palm OS requires a minimum of $200 \mathrm{~KB}$ free RAM, and Pocket PC requires a minimum of 3.0 MB free RAM. Last update, Version 2.03, November 12, 2002. 


\section{Lab Manual \\ <http://www.freewarepalm.com/medical/labmanual.shtml>}

Lab Manual is designed for medical workers who must draw and/or interpret blood labs. This free database has the most common serum labs, their drawing tubes and quantities (minimums and ideal), normal values, and clinical implications. The hyperlinked Lab Manual is easy to navigate by tapping the index letters and specific lab values to the content page. Requires the doc reader iSilo <http://www.isilo.com/>, which may be downloaded for a 30-day trial. Available on Palm OS only and uses $11 \mathrm{~KB}$ or memory. Last update, Version 3.0, October 15, 2002.

\section{Lipids by FPPalm.com <http://www.meistermed.com/MoreMeister/Lipids.htm>}

This Guide to the Management of High Cholesterol is based on the recommendations of the Third Report of the National Cholesterol Education Program (NCEP) Expert Panel on the Detection, Evaluation, and Treatment of High Blood Cholesterol in Adults (Adult Treatment Panel (ATP) III). It features recommendations for lipid management released by ATP III, evidence-based recommendations from the U.S. Preventive Services Task Force 2001 Update on Lipid Disorders in Adults, algorithm for determining 10-year risk of coronary heart disease clinical risk. Available for Palm OS only, and requires $30 \mathrm{~KB}$ of memory. Isilo reader is required.

\section{LSU ACLS 2001 Review Flash Cards <http://www.pdacortex.com/ACLS_flash_cards.htm>}

These flash cards are a review of the Advanced Cardiac Life Support (ACLS) protocols by the American Heart Association. The cards consist of 390 question and answer sets that were developed by physicians who are board certified in Emergency Medicine and Anesthesiology. The cards can be played back in sequence, by category, and randomly. Scoring is provided both as an overall grade and by category. Advanced text formatting, such as bold, italics, and bullet lists, makes it easier to read and understand. Requires the free QuizApp software (available on the same Web page) to run. Available on Palm OS only and uses 109 KB of memory; the QuizApp uses $201 \mathrm{~KB}$ of memory. Last update, May 21, 2002. 


\section{Medical Approaches <http://www.medicalapproaches.com>}

Medical Approaches was designed by junior doctors for junior doctors. This book contains approaches that help bridge the gap between problem and diagnosis and treatment, frameworks for dealing effectively with common diseases, templates for the rapid identification and management of emergencies, and a means to contribute users' own approaches and experiences with clinical problem solving. The approaches are designed to cross-refer and cut across specialty divisions and clinical settings. The hyperlinks lead users directly to individual chapters or subdivisions. A help page provides information to navigate the book. To read the book, Mobipocket reader <http://www.mobipocket.com/> is needed. The standard version of Mobipocket reader is free. Available for both Palm OS and Pocket PC and requires $1 \mathrm{MB}$ of memory.

\section{On Call-Floor Emergencies <http://pbrain.hypermart.net/medfiles.html>}

This quick reference to common hospital floor emergencies, designed to aid interns on call, was compiled by Kent Willyard, MD. It includes various categories of emergencies, their diagnoses, tests, medications, procedures, etc. Requires a doc reader to run. Available for Palm OS only, uses $15 \mathrm{~KB}$ of memory, and was released June 7, 2000.

\section{PalmEKG}

\section{<http://palmekg.com/PalmEKG/>}

The PalmEKG is an arrhythmia field guide for Palm OS PDAs, featuring 25 ECG/EKG Arrhythmias. PalmEKG includes Sinus Rhythms, Atrioventricular Blocks, Atrial Rhythms, Junctional Rhythms, and Ventricular Rhythms. Available for Palm OS only, requires Palm OS 3.0 or higher and takes $20 \mathrm{~KB}$ of memory. Version 1.0 was released in 2001.

\section{Sample Floor Notes <http://pbrain.hypermart.net/medfiles.html>}

Examples of common hospital floor notes (admission orders, op note, delivery note, etc.) for medical students and interns. A doc reader is required. Available for Palm OS only, uses 4 KB of memory, released June 7, 2000. 
Shots 2003

<http://www.immunizationed.org>

Shots 2003 is a quick reference guide to the 2003 Childhood Immunization Schedule, a collaboration of the Advisory Committee on Immunization Practices (ACIP), the American Academy of Pediatrics (AAP), and the American Academy of Family Physicians (AAFP), and the 2002-2003 Adult Immunization Schedule, recommended by the Advisory Committee on Immunization Practices (ACIP). Details on each vaccine are available by clicking on the vaccine names. Requires Palm OS 3.1 or higher and 279KB of memory. Shots 2003 runs on both color and non-color handhelds and is available on Palm OS only. Last update, January 2003.

\section{Dictionaries}

\section{Medical Abbreviations <http://www.medlists.net/Medical_Abbreviations.htm>}

The author of this application, Krystof J. Neumann, MD, has collected more than 2000 abbreviations from all medical specialties throughout his medical school training and compiled this free, easy-touse, searchable database, hoping to reduce some of the confusion that results from the use of abbreviations in medical charting. It is convenient to use a three-letter abbreviation (e.g., TLA) instead of writing out an entire phrase, but TLA may be confusing. Medical Abbreviations is unique in that it not only contains all the standard abbreviations for common disease entities, but is also the only database that was developed to specifically contain all the common shorthand abbreviations used in note writing. The author desires to distribute it free to help those medical students struggling through their clinical clerkships. It requires list.prc, a freeware that may be downloaded at <http://www.magma.ca/ $\sim \mathrm{roo} / \mathrm{list} / \mathrm{list}$.html>. Available for Palm OS only and uses $75 \mathrm{~KB}$ of memory. Last update, March 29, 2002.

\section{Medical Eponyms <http://eponyms.net>}

The Eponyms database contains 1,300 common and obscure medical eponyms (e.g., Rovsing's sign, Virchow's node) with descriptions. This is a handy reference tool for medical professionals struggling with 
hard-to-remember names for common diseases. The author of the database, Andrew Yee, a medicine senior resident at Massachusetts General Hospital, has been working on this database since he was a second-year medical student. Tap the trigger on the upper right corner to choose one of the 22 categories to find an eponym. An on-screen keyboard, with the option of switching from English letters to numbers and symbols or international letters, makes entering a name for searches much easier. The stand-alone version is for Palm OS only. There is also an alternative version of Eponyms available in a variety of databases such as JFile or Handbase format for both Palm OS and Pocket PC. Uses $114 \mathrm{~KB}$ of memory. Last update, November 12, 2002.

\section{Medical Mnemonics}

<http://www.medicalmnemonics.com/>

Medical mnemonics is a database to aid in remembering the important details. Mnemonics have existed for a long time. This software expands the circles of sharing/exchange to a larger group worldwide and ensures useful mnemonics to be available to later learners. Users may add their own new medical mnemonics via an onscreen keyboard. The stand-alone version is available for Palm OS only. There are two sizes of databases for the application. An unabridged version, which contains 1369 mnemonics, requires $85 \mathrm{~KB}$ application and $547 \mathrm{~KB}$ database, and a smaller abridged version, which contains 783 mnemonics, requires $85 \mathrm{~KB}$ application and $335 \mathrm{~KB}$ database for those with more limited space on their PDA. The AdvantGo version is available for both Palm OS and Pocket PC. The AvantGo browser is available for download from AvantGo.com for free. Last update, February 20, 2003.

\section{Medical Spanish <http://pbrain.hypermart.net/medfiles.html>}

This software contains common medical words and phrases for English-to-Spanish, including greetings, history taking, review of systems, physical examination, obstetrics/gynecology, anatomy, conditions, and medications. A pronunciation guide is included. Requires a doc file. Available for Palm OS only, uses $7 \mathrm{~KB}$ of memory, and was released on June 7, 2000. 


\section{Clinical Guidelines}

\section{ACC/AHA Pocket Guidelines <http://www.acc.org/clinical/palm_download.htm>}

The American College of Cardiology (ACC) and the American Heart Association (AHA) have developed nine pocket guidelines for Palm OS. Five of them use InfoGuide format and four of them use PDF. In the future, ACC will only develop PDF format guidelines, but will continue to maintain the InfoGuide. ACC is developing these pocket guidelines for other PDA formats, including the Pocket PC, but none is available for Pocket PC, yet. The InfoGuide format guidelines are easy to navigate. The down arrow trigger at the bottom of the page brings users to the table of contents (TOC) to choose from different chapters. Figures and tables are also listed. The InfoGuide format guidelines, their size, and release dates are as follows:

Pocket Guideline for Implantation of Cardiac Pacemakers and Antiarrhythmia Devices, 107 KB, March 2001.

Pocket Guideline for Management of Patients with Chronic Stable Angina, $153 \mathrm{~KB}, 1999$.

Pocket Guideline for The Management of Patients with Acute Myocardial Infarction, $120 \mathrm{~KB}$, November 2000.

Pocket Guideline for the Management of Patients with Unstable Angina and Non-ST-Segment Elevation Myocardial Infarction, 17 KB, August 2001.

Pocket Guideline for The Management of Patients with Valvular Heart Disease, 106 KB, December 2000.

The PDF format guidelines are also easy to navigate. The trigger at the bottom of the page leads users to the TOC, which includes chapters, tables, and figures. Users may tap and hold graphics to view them in full size. The PDF format guidelines, their size, and release dates are as follows:

Pocket Guideline for Coronary Artery Bypass Graft Surgery (CABG), 326 KB, August 2001.

Pocket Guideline for Evaluation and Management of Chronic Heart Failure in the Adult, $152 \mathrm{~KB}$, February 2002.

Pocket Guideline for the Management of Patients with Atrial Fibrillation, $225 \mathrm{~KB}$, March 2002.

Pocket Guideline for Perioperative Cardiovascular Evaluation for Noncardiac Surgery, 277 KB, 2002. 


\section{ACCP Quick Reference Guide on Antithrombotic Therapy <http://www.infoguidecenter.com/accp/>}

The American College of Chest Physicians (ACCP) Quick Reference Guide on Antithrombotic Therapy is a summary of the Sixth ACCP Consensus Conference on Antithrombotic Therapy, published in full form in Chest 119 (no 1, Suppl, January 2001). A table of contents is available for easy navigating. The document, ACCP_Guideline.pdb, is $122 \mathrm{~KB}$. It needs Word To Go, wordtogo.prc, which is $153 \mathrm{~KB}$ to run. Both files may be downloaded from the ACCP Infoguide Center for free. The Guide is currently only available for Palm OS compatible devices running Palm OS 3.0 or greater.

\section{Act in Time to Heart Attack Signs <http://hin.nhlbi.nih.gov/haac_palm/haac_palm.htm>}

This program was designed to help health care workers help patients recognize heart attack warning signs and take appropriate survival steps using guidelines from the National Heart, Lung, and Blood Institute. It includes a list of common questions and concerns, and offers responses proven effective in provider-patient interactions. It outlines the T.I.M.E. (Talk with your patients, Investigate, Make a plan, and Evaluate) method for helping patients understand and feel comfortable with the advice being given. The down arrow key at the bottom of the screen provides easy access to different sections. Available on Palm OS only, and uses $82 \mathrm{~KB}$ of memory. Released, 2001.

\section{AHA Practice Guidelines <http://www.mobilemedica.com/>}

The American Heart Association's guidelines were developed using Mobile Medica's Apprisor Viewer, an easy-to-use and easy-tonavigate tool. Apprisor is free at Mobile Medica's Web site. Currently, it is only available for Palm OS, but hopefully will be available for Pocket PC in the near future. The AHA documents, their sizes, and release dates are as follows:

AHA/ACC Guidelines for Preventing Heart Attack and Death in Patients With Atherosclerotic Cardiovascular Disease; 17 KB, (2001 Update) June 1, 2001. 
Aortic Valve Disease, 110 KB, November 1, 2002.

Atrial Fibrillation, $31 \mathrm{~KB}$, November 1, 2002.

Cardiac Pacemakers, 33 KB, November 1, 2002.

Cathflo Activase (Alteplase) Information Genentech, 82 KB, July 1, 2002.

Chest Pain, 12 KB, November 1, 2002.

Fainting, 14 KB, November 1, 2002.

Guidelines for the Management of Anaphylactoid Reactions in the Cardiac Catheterization Library; SCAI, 9 KB, Sep 1, 2000.

Heart Transplant, 33 KB, November 1, 2002.

Pulmonary Embolism and Deep Vein Thrombosis, 35 KB, November 1, 2002.

Restenosis: Repeat Narrowing of a Coronary Artery, $70 \mathrm{~KB}$, November 1, 2002.

Smoking Cessation Strategies, 20 KB, November 1, 2002.

Statins: Powerful Drugs for Lowering Cholesterol, $57 \mathrm{~KB}$, November $1,2002$.

Take Heart With Heart Failure, 23 KB, November 1, 2002.

The Implantable Cardioverter-Defibrillator, 47 KB, November 1, 2002.

Warning Signs of a Heart Attack, 13 KB, November 1, 2002.

\section{ATP III Cholesterol Management Guidelines <http://hin.nhlbi.nih.gov/atpiii/atp3palm.htm>}

As part of the educational mission of the National Cholesterol Education Program of the National Institutes of Health (NIH) National Heart, Lung, and Blood Institute (NHLBI), the Palm OS program for application of the Third Report of the National Cholesterol Education Program (NCEP) Expert Panel on Detection, Evaluation, and Treatment of High Blood Cholesterol in Adults (Adult Treatment Panel III, ATP III) is made available. This interactive guideline tool will assist the clinician in implementing the ATP III Cholesterol Guidelines at the point of care. It also contains usable information from ATP III, including ATP III classification of lipid levels, ATP III Coronary Heart Disease (CHD) risk assessment, therapeutic Lifestyle Changes (TLC), drug therapy for lipid lowering, information on the metabolic syndrome, and issues for special populations. A freeware "MathLib.prc" must also be installed on the Palm OS device for the ATP3 Cholesterol Management Implementation Tool to work correctly. This program is easy to use. By tapping the space for value, a pop-up window comes out with a numeric 
and alphabetic keypad. Tap the numbers to enter the value to have the risk factor calculated. Available for Palm OS only and uses $123 \mathrm{~KB}$ of memory. Version 1.0 was released 2001.

\section{COPD Guidelines Tool for Palm OS <http://hin.nhlbi.nih.gov/copd.htm>}

This is a practical point-of-care reference to the Pocket Guide to COPD Diagnosis, Management, and Prevention from the Global Initiative for Chronic Obstructive Lung Disease. It is easy to tap the down arrow keys to navigate from its four categories: assess and monitor COPD, reduce COPD risk factors, management of stable COPD, management of acute COPD exacerbations and their subcategories. Clinicians are advised to be thoroughly familiar with the full GOLD Executive Summary report before using this reference tool in patient care. It is on Palm OS only and uses $106 \mathrm{~KB}$ of memory. Released, 2001.

\section{The NHLBI/NAEPP Guidelines} <http://hp2010.nhlbihin.net/as_palm.htm>

The NHLBI/NAEPP Guidelines for Palm OS is a succinct and practical point-of-care reference to the full NAEPP Expert Panel Report 2: Guidelines for the Diagnosis and Management of Asthma. The content includes initial diagnosis and assessment, control of contributing factors, pharmacologic treatment, treatment of exacerbations, medication dosages, and patient education. TealInfo software <http://www.tealpoint.com/ softinfo.htm>, which allows a 30-day free trial, must be installed to use the NHLBI/NAEPP Guidelines. Available on Palm OS only, and uses 50 KB of memory. Version 1.0, August 2000.

\section{OEI Treatment Guidelines Implementation Tool for Palm OS <http://hin.nhlbi.nih.gov/obgdpalm.htm>}

The Obesity Education Initiative (OEI) Guidelines for Palm OS is intended as a point-of-care reference to the full "Clinical Guidelines on the Identification, Evaluation, and Treatment of Overweight and Obesity in Adults: The Evidence Report" <http://www.nhlbi.nih.gov/guidelines/ obesity/ob_gdlns.htm> and the "Practical Guide to the Identification, Evaluation, and Treatment of Overweight and Obesity in Adults" <http://www.nhlbi.nih.gov/guidelines/obesity/practgde.htm>. Clinicians are advised to be thoroughly familiar with these documents before us- 
ing this reference tool in patient care. This simple, easy-to-use tool's interactive design allows the use of a built-in keypad to enter a value and instantly generate individualized and evidence-based assessment and treatment recommendations for each patient. Features include body mass index calculation, assessment of cardiovascular disease risk factors, determination of need for treatment, individualized weight loss goals, individualized treatment recommendations (lifestyle therapy, pharmacotherapy, and surgery options), follow-up and maintenance recommendations, and evidence-based supporting information available throughout. Available for Palm OS only and uses $95 \mathrm{~KB}$ of memory. Version 1.0, 2000.

Received: January 14, 2003

Revised: February 12, 2003

Accepted: March 7, 2003

\section{REFERENCES}

1. Zaroukian, M.H., and Sousa, A. "Handheld Computing in Resident Education: Benefits, Barriers, and Considerations." Seminars in Medical Practice 5(4, 2002): 33-44.

2. Embi, P.J. "Information at Hand: Using Handheld Computers in Medicine." Cleveland Clinic Journal of Medicine 68(10, 2001): 840-6, 848.

3. "Taking the Pulse: Physicians and Emerging Information Technologies: Seven Key Market Trends in 2003." December 11, 2002. Available: <http://manhattanresearch. com/Taking\%20the\%20Pulse\%20(121102)\%20press\%20release.pdf $>$ Accessed: January $25,2003$. 


\section{APPENDIX}

PDA Medical Software Web Resources

American Academy of Family Physician Medical Files/Applications $<$ http://www.aafp.org/x472.xml>

CollectiveMed <http://collectivemed.com/>

Document readers at MemoWare $\langle$ http://www.memoware.com/mw.cgi/?screen=help_format $>$

Ectopic Brain <http://pbrain.hypermart.net/>

FreewarePalm.com <http://freewarepalm.com>

Handheld Computing Resources at Informatics Review <http://www.informatics-review.com/handheld.html>

Handheldmed <http://www.handheldmed.com/>

Healthy PalmPilot <http://www.healthypalmpilot.com/>

Medical Politeer <http://www.medicalpiloteer.com/>

Medical Pocket PC <http://medicalpocketpc.com/>

MedLists <http://www.medlists.net/>

New Media Medicine

<http://www.newmediamedicine.com/mobile/software.htm>

PdaMD <http://pdamd.com/>

PDAs for Health Care Providers at Arizona Health Sciences Library $<$ http://educ.ahsl.arizona.edu/pda/index.htm>

Skyscape <http://www.skyscape.com/> 